and approximation may be accomplished where only the connective tissue of the cicatrised wound is interposed. In all this, investigation and experiment lag behind clinical experience, and while it is known that function can be restored after the excision of even a considerable portion of a nerve trunk, the mode in which this regeneration is brought about has yet to be determined.

Newcastle-on-Tyne.

\section{ON LENTICONUS.}

By LAWFORD KNAGGS, M.C. CANTAD, F.R.C.S., OPHTHALMIC SURGEON TO THE LEEDS PUBLIC DISPENSARY.

"LENTICONUS" is the term used to describe a peculiar alteration in the shape of the crystalline lens, which consists, as the name implies, in the formation of a conical protrusion from its surface. It may be that in some instances the abnormality is congenital, but it is certain that in others the defect is developed during life. In the Archives of Ophthalmology (1889, vol. xviii., p. 451) Dr. H. Knapp of New York has given abstracts of the three previously recorded cases, and has added a fourth. For the purposes of this article the main features of those cases are here briefly recapitulated.

1. D. Webster.-Male, aged twenty-four. Oblique illumination showed'a well-marked conical projection in the central part of the anterior capsule. "There was a small posterior cataract, and the whole posterior capsule was dotted over with very minute opacities, radiating from the posterior pole of the lens. The affection was alike in both eyes, and if it was not congenital it certainly was acquired in early youth." The patient "could read J. 1 from a distance close by up to 3 in., with $-\frac{1}{2}$ " and a cylinder $=\mathrm{V}=\frac{20}{200}$ in each eye.

2. Van der Laan and Placidus.-Male, aged twenty-three. Anterior lenticonus producing high myopia, whilst the peripheral part of the lens showed $\mathrm{H}=3 \mathrm{I}$ ). The condition was said to have existed eight years, and developed gradually. No statement as to whether one or both eyes were affected. 2

3. F. Meyer.-Boy, aged ten. Left eye normal. Right eye had squinted outwards for three or four years, but defective sight had been accidentally discovered only nine months previously. "A conical elevation was seen to project from the posterior pole of the lens into the vitreous, bearing on and around its apex three white little patches" (posterior polar eataract). Vision improved by $-12 \mathrm{D}^{3}$

4. H. Knapp.-Healthy girl, aged eight. Left eye normal. The sight of the right eye was found defective only on examination by an oculist. Fingers at 12 feet, with $-\frac{1}{13}$, sight $=\frac{20}{200}$. Lenticonus posterior and central. By focal illumination the abnormality could not be detected. No posterior polar cataract; whether congenital or acquired, stationary or progressive, was uncertain.

The following case, which differs in some important points from the foregoing, is at present attending amongst my outpatients.

E. W-_ aged sixty-five, had good vision till six months ago. Her daughter stated that it would be four years since she astonished her family by reading a newspaper pasted upon a wall about a yard and a half away from her. At Christmas, 1890, her attention was directed to her sight because she was unable to recognise the minister at chapel or people in the street. She could see to sew and read as well as ever. She first sought advice on April 27 th, 1891. $0 \mathrm{n}$ external examination there was nothing abnormal except a greenish haze of both lenses. Vision : Right eye $=\frac{6}{\mathrm{nll}}$ $c-10 \mathrm{D}$, and $-1 \mathrm{D}$ cyl. $=\frac{6}{12}$ fairly. Left eye $=\frac{6}{\text { nil, }} c-$ $11 \mathrm{D}=\frac{6}{18}$. She read J. 8 at a distance of 4 or 5 in. without glasses. There was metamorphopsia, smaller objects being multiplied or distorted, but larger ones confused. On dilating

Archives of Ophthalmology and Otology, rol. iv., p. 382

2 Abstract in Nagel's Jahresbericht, 1880, p. 369 . Period. de Oftalm. Prat., Rivista bim., Lisbon, No. 3, 1880

3 Hirschberg's Centralblatt f. Aug., Bd, xii., p. 41 the pupils with homatropine the following pathological condition was found to be present, and equally advanced in both eyes. The cornea was of normal curvature and perfectly healthy, except for a faint but complete arcus senilis. On focal illumination in the centre of the pupil there was a greenish-yellow circular opacity sulliciently defined to be measured (diameter $=4.5 \mathrm{~mm}$.). It was most dense at the centre, but at its periphery blended rapidly with the greenish yellow tinge of the lens substance. The appearance was not very unlike that presented by nuclear cataract. for which by this method of examination it could have been mistaken, but the opacity was evidently at the posterior surface of the lens. On examining with the ophthalmoscope at a distance of three or four feet by the direct method, a clear uniform red reflex was obtainable with slight manipulation of the mirror, dark shadows could be made to play round the central point of the lens, but always leaving a narrow zone of red reflex between the periphery of the shadow and the margin of the pupil. Approaching to about twelve inches distant, a dise with a diameter of $5.5 \mathrm{~mm}$. was seen centrally placed in the pupil; it was of a slightly duller red than the peripheral part of the reflex, from which it was marked off by a faint circular shadow, which varied somewhat on movement of the mirror, and sometimes took a reddish tinge, due to the retinal vessels. The shadows already deseribed as circling round the central point of the lens were formed eatirely within this disc, and a nore careful observation showed that they were similar to those formed by kerato. scopy in a myopic eye--viz., moving with the mirror,--but instead of the edge of the shadow being straight is was crescentic, and the two ends of the crescent embraced the centre of the disc. Opacities were seen floating in the vitreous, and they sometimes appeared to be deflected from a straight descent, and to fall along the edge of the disc. Kaleidoscopic novements of the vessels, similar to those seen in cases of conical cornea, were very aistinetly seen within the dise, and on the observer moving his head the general muvement of the vessels was in a direction opposite to that in which he moved. Oatside the disc the vessels were not visible. With the ophthalmoscope close up the disc offered in some positions a distinct bar to observation; but in others, with the help of a strong minus lens, the details of the fundus could be clearly seen without any diffieulty, but more or less altered. Kaleidoscopic movements of vessels were best seen when looking at the optic papilla; but if a single vessel was watched the central part of the observed portion could be seen to change from a concavity to a convexity, as the observer's eye was moved from side to side. Though the vitreous contained opacities it was not abnormally fluid. The fundus was healthy. Besides the defect of sight, she complained greatly of pain in the nape of her neck, for which there was no obvious cause, and she suffered from oxaluria. (Urine: sp. gr. 1015, acid ; no albumen, no sugar, no phosphates. Microscope : numerous oxalates and a few masses in the shape of casts, and composed of small oxalates: some pus cells, and vaginal and bladder epithelium.) She had been deaf since she was twenty-seven. The other organs were healthy. She was ordered $-7 \mathrm{D}$ for all purposes, and with the improvement in distant vision thus obtained she is quite satisfied.

In the Ophthalmological Society's Transactions for 1889 , vol. ix., p. 113, Mr. R. W. Doyne describes "a peculiar form of lens degeneration." The description of the condition is very similar to that already given of the case of $\mathrm{E} . \mathrm{W}$ The patient was a woman, aged seventy, whose sight had been failing for some years. Focal illumination produced "the appearance of quite a dense nuclear opacity," but " the nucleus of the lens offered no interference to the passage of the rays of light through it, bat around it there could be seen a shifting shadow such as is observed in cases of conical cornea." There was a sharply defined difference of $11 \mathrm{D}$ between the nucleus and the cortex, the refraction of the cortex being $-3 \mathrm{D}$, and that of the nucleus $-14 \mathrm{D}$, with which lens $\mathrm{V}=\frac{6}{36}$. In the general index for vols. $i$. to ix. this case is described as "lenticonus." Mr. Hartridge, on page 115 of the same volume, iefers to a young lady, aged seventeen, whose lenses were perfectly transparent on oblique illumination, "but on looking at them with a concave mirror it was seen that the lens was composed of two distinct parts, the central nuclear part looking like a large globule of oil." "The refraction through 
the centre of the lens was $5 \mathrm{D}$ stronger than the refraction through the margin." The condition was congenital.

But little appears to be known of the pathology of this condition. Professor $O$. Becker of Heidelberg examined the eye of a rabbit, in which the lens showed a conical protrusion in the region of the posterior pole, and in which apparently healthy lens fibres were covered with normal lens capsule." In three cases (Van der Laan's, D yyne's, and the writer's) the disease was nndoubtedly acquired; in the others it appeared at so early an age that a suspicion of congenital origin was raised. Its occurrence in one eye only makes it probable that it may be traced in certain instances to some purely local defect, but when both eyes are simultaneously affected a general constitutional cause is to be suspected, and in E. W. the oxaluria was probably an expression of general deterioration of health. There is sufficient evidence in the recorded cases to justify

FIG. 1.

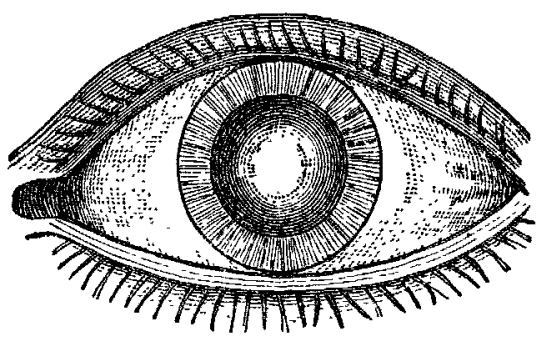

The central opacity seen on oblique illumination. The depth of the opacity from the anterior surface of the lens is not well shown.

FIG. 2.

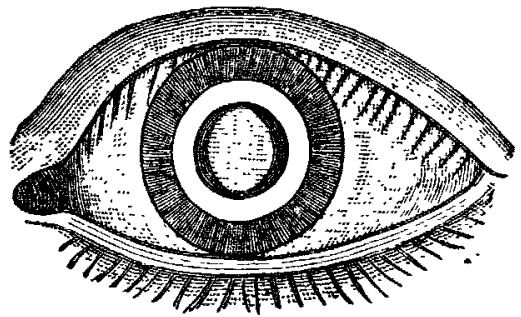

The oil-globule-like disc seen by transmitted light.

Fig. 3.

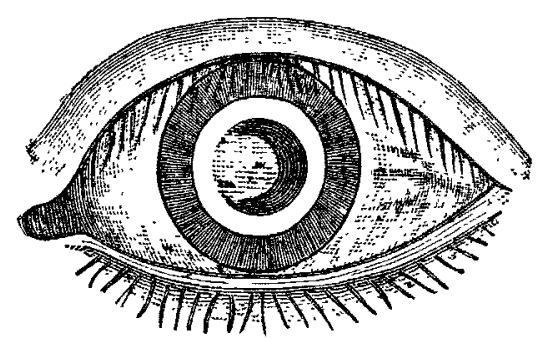

The crescentic shadow, produced by keratoscopy, within the disc.

('The above sketches are slightly diagrammatic.)

the assumption that the immediate cause of lenticonus is a weakening of the lens capsule, rendering it unequal to restrain the natural elasticity of the lens substance. In every instance the cone has developed at the centre of either the anterior or posterior surface of the lens. Here, where the lens is thickest, its elasticity is probably greatest, and the capsule is likely to be weaker because the poles are furthest away from the circumference of the lens, where the capsule is strengthened by fusion with the fibrillar elements of the suspensory ligament. The occasional coexistence of posterior polar cataracts (two cases) is in favour of this assumption. It is true that in Webster's case the cones were anterior, and the opacities affected the whole of the posterior surfaces; but the inference is not unfair that both conditions pointed to the same thing-viz, a diseased capsule. It is easier to explain lenticonus by a defective capsule than by the supposition of some morbid change in the lens fibres. For the latter hypothesis it would be essen.

Knapn's paper: Zur Anatomie der Gesunden u. Kranken Linse, p. 126. Wiesbaden: J. F. Bergmann. tial to assume a change of such a nature that would not obstruct the transmission of light, and would lead to a swelling of the lens in the situations in which lenticonus is always oluserved. Both suppositions are contrary to what we should anticipate if the lens substance were really diseased; and Professor Becker's observation gives them no support. If the explanation suggested here is the true one, we should expect the condition to be progressive only up to a certain point. However weak the capsule might be, the protrusion would cease to increase as som as the lens sub. stance had expanded to the full extent of its elasticity, while intermediate stages might result from the retention by the capsule of a variable amount of restraining power. In the cases referred to above there is nothing opposed to this view, whilst the length of time that the condition had existed in the first two cases would favour it

The treatment of lenticonus will vary with the particular case. When one eye only is affected and the other eve is good it may be wiser to leave it alone. In early life, when the condition is symmetrical and calls for treatment, the lenses may be removed by discission or suction. In later life, and where the condition is acquired as a consequence of impaired health, it may be best to palliate with glasees rather than run the obviously greater risks of removal of both lenses in their capsules. This is the plan I have adopted with E. W- In her case the metamorphopsia is the only symptom which would suggest a more radical treatment, and it is so slight a trouble to her that to expose her to the risks of an operation would be undesirable.

In conclusion, the points which enable a diagnosis of lenticonus to be made, when the cone cannot be rendered visible by focal illumination, are the following:-l. The oil-globule. like disc. 2. The great difference of refraction between the margin of the lens and the central portion, the latter being always highly myopic. 3 The kaleidoscopic movements of the retinal vessels. 4. The exclusion of conical cornea.

Leeds.

\section{A CASE OF "ANTHRAX" SUCCESSFULLY TREATED BY EXCISION AND MER CURIAL IRRIGATION.}

By J. A. HUTTON, M.B., B S., M.R.C.S., L.R.C.P.

As cases of "anthrax" or "malignant pustule" are of such rare occurrence that the majority of practitioners pass through a lifelong active practice without being called on to deal with a case, and as severe cases are so very fre. quently fatal, I thought it would interest the readers of ThE LANCET to have the following successful case brought before them.

J. H-, a stout, ruddy-faced, well-knit, healthy. looking man, a veterinary surgeon by profession, practising in a village a few miles from Scarborough, on the night of Feb. 11th, 189l, was called to see an Irish beifer suffering from what was diagnosed as, and subsequently proved to be by post-mortem examination, splenic apoplexy. On his arrival the animal was dead, and the owner was anxious the carcase should be bled, so that it might be sold in the usual way. The animal being dead, the division of the smaller vessels failed to remove the blood; therefore an incision was made into the thorax and the aorta divided. The carcase was next placed in various positions to facilitate the flow of blood. J. H- was assisted by R. P— and G. B-, and during their manipulations with the carcase all three managed to scratch themselves slightly. The subsequent history of these two assistants is extremely interesting. On Feb. 15th R. P began to feel ill, with extreme swelling of the arm and an angry "lump" at its lower part. Despite the vigorous treatment by the village surgeon, he grew gradually worse, and died on the 19th, exhibiting all the signs and symptoms of a severe attack of anthrax. On the 2lst G. B- became affected with great swelling of the inoculated arm and pyrexia. The arm was incised and cauterised by the local surgeon, with the result that he has made a slow but complete recovery. On the 17th J. H- noticed a slight papule at the site of inoculation on the lower part of the right forearm, and felt very ill. with high fever, loss of appetite, swelling of the limb, and pain. The papule was surrounded by a zone of inflamed 